\title{
A NOTE ON DUAL INTEGRAL EQUATIONS INVOLVING INVERSE ASSOCIATED WEBER-ORR TRANSFORMS
}

\author{
NANIGOPAL MANDAL \\ Calcutta Mathematical Society \\ AE-374, Sector-I, Salt Lake City \\ Calcutta 700 064, INDIA \\ B. N. MANDAL \\ Physical and Earth Sciences Division \\ Indian Statistical Institute \\ 203, B.T. Road, Calcutta 700 035, INDIA
}

(Received July 2, 1992 and in revised form March 30, 1993)

\begin{abstract}
We consider dual integral equations involving inverse associated Weber-Orr transforms. Elementary methods have been used to reduce dual integral equations to a Fredholm integral equation of second kind. Some known results are obtained as special case.
\end{abstract}

KEY WORDS AND PHRASES: Dual integral equations, Associated Weber-Orr transform, Fredholm integral equation.

1980 AMS SUBJECT CLASSIFICATION CODES: 44A20.

\section{INTRODUCTION}

Dual integral equations involving inverse associated Weber-Orr transforms $W_{\mu, \nu}^{-1}[;]$, arise in the study of mixed boundary value problems in elasticity and torsion. Srivastav $[1,2]$ considered some dual integral equations involving $W_{v, r}^{-1}[;]$ and $W_{0,0}^{-1}[;]$. In a recent paper, Nasim [3] considered the following dual integral equations:

$$
\begin{array}{ll}
W_{v-k, \nu}^{-1}\left\lfloor\xi^{-2 \alpha} \psi(\xi) ; x\right]=f_{1}(x), & a \leq x \leq c, \\
W_{v-k, \downarrow}^{-1}\left[\xi^{-2 \beta} \psi(\xi) ; x\right]=-f_{2}(x), & c<x<\infty,
\end{array}
$$

where $k=1,2, \ldots, v>-1$ and $\psi$ is an unknown function. A general solution with a few special cases was established in [3] by using elementary methods. However, the analysis in these solutions is rather complicated.

In the present note we have considered the following dual integral equations

$$
\begin{array}{ll}
W_{v-\gamma, \nu}^{-1}\left[\xi^{-2 \alpha} \psi(\xi), x\right]=g_{1}(x), & a \leq x \leq c, \\
W_{v-\gamma, \nu}^{-1}\left[\xi^{-2 \beta} \psi(\xi), x\right]=g_{2}(x), & c<x<\infty,
\end{array}
$$

where $\nu>-\frac{1}{2}, \gamma(>0)$ is not an integer with $\gamma+\alpha-\beta$ a positive integer, $\psi$ is an unknown function. This system is reduced to the solution of a Fredholm integral equation of second kind for $-1<\alpha-\beta<1$ with $\alpha-\beta \neq 0$. The method utilized herc involves the use of certain multiplying factors to the equations somewhat similar to the method used by Noble [4], Mandal [5] and the references cited therein in connection with dual integral equations with Bessel function Kernel. 
The analysis throughout this paper is purely formal and the change of order of integrations in various double integrals are assumed to be justifiable.

\section{SOME USEFUL RESULTS}

We state below, for convenience, some results and definition which will be used subsequently in the naper. Let,

$$
R_{\mu \nu}(x ; \xi, a)=J_{\mu \prime}(x \xi) Y_{\nu}(\xi a)-Y_{\mu}(x \xi) J_{\nu}(\xi a) .
$$

Using the formulae (33), p. 26 and (35), p. 103 of [6], it may be deduced that

$$
\begin{aligned}
\int_{a}^{t} x^{1+\mu}\left(t^{2}-x^{2}\right)^{\alpha} R_{\mu \nu}(x ; \xi, a) d x= & 2^{\alpha} \Gamma(\alpha+1) \xi^{-\alpha-1} t^{\mu+\alpha+1} R_{\mu+\alpha+1, v}(t ; \xi, a) \\
& -\frac{2^{\mu+1}}{\pi} \xi^{-\alpha-1} t^{\mu+\alpha+1} \Gamma(\mu+1) J_{v}(a \xi) S_{\alpha-\mu, \alpha+\mu+1}(t \xi) \\
& -\int_{0}^{a} x^{1+\mu}\left(t^{2}-x^{2}\right)^{\alpha} R_{\mu, \nu}(x ; \xi, a) d x,
\end{aligned}
$$

for $\operatorname{Re}(\alpha)>-1, \operatorname{Re}(\mu)>-1, a>0, S_{\mu, v}(z)$ being Lommel's function. From the formulae (32), p. 25 and (38), p. 104 of [6], it may also be deduced that

$$
\int_{1}^{\infty} x^{1-\mu} R_{\mu, v}(x ; \xi, a)\left(x^{2}-t^{2}\right)^{\beta} d x=2^{\beta} \Gamma(\beta+1) t^{1+\beta-\mu \xi} \xi^{-\beta-1} R_{\mu-\beta-1, v}(t ; \xi, a),
$$

for $\operatorname{Re}(\beta)>-1$ and $\operatorname{Re}(\mu-2 \beta)>\frac{1}{2}$.

DEFINITION. We define,

$$
\begin{aligned}
& W_{\mu, v}[g(x) ; \xi]=\int_{a}^{\infty} x g(x) R_{\mu, v}(x ; \xi, a) d x, \quad 0<\xi<\infty, a>0, \\
& W_{\mu, v}^{-1}[\bar{g}(\xi) ; x]=\int_{0}^{\infty} \frac{\xi \bar{g}(\xi) R_{\mu, v}(x ; \xi, a)}{J_{v}^{2}(\xi a)+Y_{v}^{2}(\xi a)} d \xi,
\end{aligned}
$$

where $R_{\mu, v}(x ; \xi, a)$ is defined by (1).

LEMMA. If $0<k<\frac{1}{2} v+\frac{3}{4}, k=1,2,3, \ldots, v>-\frac{1}{2}$ and $x^{k+\frac{1}{2}} g(x)$ is summable in the infinite interval $(a, \infty)$, then

$$
\begin{aligned}
& \bar{g}(\xi)=W_{v-k, v}[g(x) ; \xi], \\
& g(x)=W_{v-k, v}^{-1}[\bar{g}(\xi) ; x] .
\end{aligned}
$$

This result is proved in [7].

\section{REDUCTION OF DUAL INTEGRAL EQUATION TO A FREDHOLM INTEGRAL EQUATION OF SECOND KIND}

We consider the dual integral equations

$$
\int_{0}^{\infty} \xi^{-2 \alpha+1} \frac{R_{v-\gamma, v}(x ; \xi, a)}{J_{v}^{2}(\xi a)+Y_{v}^{2}(\xi a)} \psi(\xi) d \xi-g_{1}(x), a \leq x \leq c,
$$




$$
\int_{0}^{\infty} \xi^{-2 \beta+1} \frac{R_{v-\gamma, v}(x ; \xi, a)}{J_{v}^{2}(\xi a)+Y_{v}^{2}(\xi a)} \psi(\xi) d \xi=g_{2}(x), \quad c<x<\infty,
$$

where $R_{\mu, v}(x ; \xi, a)$ is defined by (1), $v>-\frac{1}{2}, \gamma$ is positive non-integer with $v-\gamma>-1$ and $-1<\alpha-\beta<1, \alpha-\beta \neq 0$ with $\gamma+\alpha-\beta$ a positive integer.

Multiplying each side of (4) by $x^{1+v-\gamma}\left(t^{2}-x^{2}\right)^{\alpha^{\prime}}$ where $\alpha^{\prime}>-1$ and integrating with respect to $x$ from $a(>0)$ to $t$, then interchanging orders of integration on the left hand side and using (2), we obtain

$$
\begin{aligned}
& \int_{0}^{\infty} \xi^{-2 \alpha-\alpha^{\prime}} \frac{R_{v-\gamma+\alpha^{\prime}+1, v}(t ; \xi, a)}{J_{v}^{2}(\xi a)+Y_{v}^{2}(\xi a)} \psi(\xi) d \xi=\frac{2^{-\alpha^{\prime}}}{\Gamma\left(\alpha^{\prime}+1\right)} t^{-v+\gamma-\alpha^{\prime}-1} \int_{a}^{t} x^{1+v-\gamma}\left(t^{2}-x^{2}\right)^{\alpha^{\prime}} g_{1}(x) d x \\
& +\frac{2^{v-\gamma-\alpha^{\prime}+1}}{\pi \Gamma\left(\alpha^{\prime}+1\right)} \Gamma(v-\gamma+1) \int_{0}^{\infty} u^{-2 \alpha-\alpha^{\prime}} \frac{S_{\alpha^{\prime}-v+\gamma, \alpha^{\prime}+v-\gamma+1}(t u)}{J_{v}^{2}(u a)+Y_{v}^{2}(u a)} J_{v}(u a) \psi(u) d u \\
& +\frac{2^{-\alpha^{\prime}}}{\Gamma\left(\alpha^{\prime}+1\right)} t^{-v+\gamma-\alpha^{\prime}-1} \int_{0}^{\infty} \frac{u^{-2 \alpha+1} \psi(u)}{J_{v}^{2}(u a)+Y_{v}^{2}(u a)}\left[\int_{0}^{a} \rho^{1+v-\gamma}\left(t^{2}-\rho^{2}\right)^{\alpha^{\prime}} R_{v-\gamma, v}(\rho ; u, a) d \rho\right] d u,
\end{aligned}
$$

for $a \leq t \leq c, \alpha^{\prime}>-1, v>\gamma-1$.

Similarly, multiplying each side of (5) by $x^{1-v+\gamma}\left(x^{2}-t^{2}\right)^{\beta^{\prime}}$ where $\beta^{\prime}>-1$ and integrating with respect to $x$ from $t$ to $\infty$, then interchanging orders of integration and using (3), we obtain

$$
\int_{0}^{\infty} \xi^{-2 \beta-\beta^{\prime}} \frac{R_{v-\gamma-\beta^{\prime}-1, v}(t ; \xi, a)}{J_{v}^{2}(\xi a)+Y_{v}^{2}(\xi a)} \psi(\xi) d \xi=\frac{2^{-\beta^{\prime}}}{\Gamma\left(\beta^{\prime}+1\right)} t^{v-\gamma-\beta^{\prime}-1} \int_{1}^{\infty} x^{1-v+\gamma}\left(x^{2}-t^{2}\right)^{\beta^{\prime}} g_{2}(x) d x,
$$

for $c<t<\infty,-1<\beta^{\prime}<\frac{1}{2}\left(v-\gamma-\frac{1}{2}\right)$.

In (6) and (7), we should like to set

$$
\nu-\gamma+\alpha^{\prime}+1=\nu-\gamma-\beta^{\prime}-1 \text { and }-2 \alpha-\alpha^{\prime}=-2 \beta-\beta^{\prime}
$$

so that the orders of $R_{\mu, v}(t ; \xi, a)$ and the powers of $\xi$ would be identical. This would mean, on solving these equations, that

$$
\alpha^{\prime}=-1-(\alpha-\beta), \quad \beta^{\prime}=-1+(\alpha-\beta) .
$$

Since $\alpha^{\prime}$ and $\beta^{\prime}$ must be greater than -1 this is impossible. To overcome this difficulty we consider the following two cases separately.

Case (i). $-1<\alpha-\beta<0, \alpha-\beta<\min \left\{\frac{1}{2}\left(\nu-\gamma-\frac{1}{2}\right), \frac{1}{2}\left(\nu-2 \gamma+\frac{3}{2}\right)\right\}$.

We take $\alpha^{\prime}=-1-(\alpha-\beta), \beta^{\prime}=\alpha-\beta$. Because of the conditions on $\alpha^{\prime}, \beta^{\prime}$ in (6) and (7), we must have $-1<\alpha-\beta<0, \alpha-\beta<\frac{1}{2}\left(\nu-\gamma-\frac{1}{2}\right)$. Then (6) and (7) gives

$$
\begin{aligned}
& \int_{0}^{\infty} \xi^{1-\alpha-\beta} \frac{R_{v-\gamma-\alpha+\beta, v}(t ; \xi ; a)}{J_{v}^{2}(\xi a)+Y_{v}^{2}(\xi a)} \psi(\xi) d \xi=\frac{2^{1+\alpha-\beta}}{\Gamma(\beta-\alpha)} t^{-v+\gamma+a-\beta} \int_{a}^{t} x^{1+v-\gamma}\left(t^{2}-x^{2}\right)^{\beta-\alpha-1} g_{1}(x) d x \\
& \quad+\frac{2^{v-\gamma+\alpha-\beta+2}}{\pi \Gamma(\beta-\alpha)} \Gamma(v-\gamma+1) \int_{0}^{\infty} u^{1-\alpha-\beta} \frac{S_{-v+\gamma-\alpha+\beta-1, v-\gamma-\alpha+\beta}(t u)}{J_{v}^{2}(u a)+Y_{v}^{2}(u a)} J_{v}(u a) \psi(u) d u \\
& +\frac{2^{1+\alpha-\beta}}{\Gamma(\beta-\alpha)} t^{-v+\gamma+\alpha-\beta} \int_{0}^{\infty} \frac{u^{-2 \alpha+1} \psi(u)}{J_{v}^{2}(u a)+Y_{v}^{2}(u a)}\left[\int_{0}^{a} \rho^{1+v-\gamma}\left(t^{2}-\rho^{2}\right)^{\beta-\alpha-1} R_{v-\gamma, v}(\rho ; u, a) d \rho\right] d u,
\end{aligned}
$$


for $a \leq t \leq c$, and

$$
\begin{gathered}
\int_{0}^{\infty} \xi^{-\alpha-\beta} \frac{R_{v-\gamma-\alpha+\beta-1, v}(t ; \xi, a)}{J_{v}^{2}(\xi a)+Y_{v}^{2}(\xi a)} \psi(\xi) d \xi=\frac{2^{\beta-\alpha}}{(1+\alpha-\beta)} t^{v-\gamma-\alpha+\beta-1} \int_{1}^{\infty} x^{1-v+\gamma}\left(x^{2}-t^{2}\right)^{\alpha-\beta} g_{2}(x) d x, \\
c<t<\infty .
\end{gathered}
$$

Now multiply both sides of (9) by $t^{-\nu+\gamma+\alpha-\beta+1}$ and differentiate with respect to $t$, then using the relation

$$
\frac{1}{t} \frac{d}{d t}\left[t^{-\mu} R_{\mu, v}(t ; \xi, a)\right]=-t^{-\mu-1} \xi R_{\mu+1, v}(t ; \xi, a)
$$

we obtain

$$
\begin{gathered}
\int_{0}^{\infty} \xi^{1-\alpha-\beta} \frac{R_{v-\gamma-\alpha+\beta, v}(t ; \xi, a)}{J_{v}^{2}(\xi a)+Y_{v}^{2}(\xi a)} \psi(\xi) d \xi=-\frac{2^{\beta-\alpha}}{\Gamma(1+\alpha-\beta)} t^{\nu-\gamma-\alpha+\beta-1} \frac{d}{d t} \int_{t}^{\infty} x^{1-v+\gamma}\left(x^{2}-t^{2}\right)^{\alpha-\beta} g_{2}(x) d x, \\
c<t<\infty .
\end{gathered}
$$

Equations (8) and (10) have identically the same left-hand side which is therefore defined for all $t \geq a$. Since we have assumed $\gamma+\alpha-\beta$ to be a positive integer and $\alpha-\beta<\frac{1}{2}\left(v-2 \gamma+\frac{3}{2}\right)$, therefore, by the lemma in section 2 , we find

$$
\begin{aligned}
\psi(\xi)= & \xi^{\alpha+\beta}\left[\frac{2^{1+\alpha-\beta}}{\Gamma(\beta-\alpha)} \int_{a}^{c} t^{1-v+\gamma+\alpha-\beta} R_{v-\gamma-\alpha+\beta, v}(t ; \xi, a) G_{1}(t) d t\right. \\
& +\frac{2^{\beta-\alpha}}{\Gamma(1+\alpha-\beta)} \int_{c}^{\infty} t^{\nu-\gamma-\alpha+\beta} R_{v-\gamma-\alpha+\beta, v}(t ; \xi, a) G_{2}(t) d t \\
& \left.+\frac{2^{1+\alpha-\beta}}{\Gamma(\beta-\alpha)} \int_{0}^{\infty} u^{1-\alpha-\beta} K(u, \xi) \psi(u) d u\right],
\end{aligned}
$$

where

$$
\begin{aligned}
G_{1}(t) & =\int_{a}^{t} x^{1+v-\gamma}\left(t^{2}-x^{2}\right)^{\beta-\alpha-1} g_{1}(x) d x, \\
G_{2}(t) & =-\frac{d}{d t} \int_{t}^{\infty} x^{1-v+\gamma}\left(x^{2}-t^{2}\right)^{\alpha-\beta} g_{2}(x) d x, \\
K(u, \xi) & =\frac{\left(\frac{1}{\pi}\right) 2^{1+v-\gamma} \Gamma(1+v-\gamma) J_{v}(u a) F_{1}(u, \xi)+u^{-\alpha+\beta} F_{2}(u, \xi)}{J_{v}^{2}(u a)+Y_{v}^{2}(u a)}, \\
F_{1}(u, \xi) & =\int_{a}^{c} t S_{-v+\gamma-\alpha+\beta-1, v-\gamma-\alpha+\beta}(t u) R_{v-\gamma-\alpha+\beta, v}(t ; \xi, a) d t, \\
F_{2}(u, \xi) & =\int_{a}^{c} t^{1-v+\gamma+\alpha-\beta} K_{1}(u, t) R_{v-\gamma-\alpha+\beta, v}(t ; \xi, a) d t, \\
K_{1}(u, t) & =\int_{0}^{a} \rho^{1+v-\gamma\left(t^{2}-\rho^{2}\right)^{\beta-\alpha-1} R_{v-\gamma, v}(\rho ; u, a) d \rho .}
\end{aligned}
$$

Equation (11) is a Fredholm integral equation of second kind. 
Case (ii). $0<\alpha-\beta<1, \alpha-\beta<\frac{1}{2}\left(v-\gamma+\frac{3}{2}\right)$.

In this case, we choose $\alpha^{\prime}=\beta-\alpha, \beta^{\prime}=-1+\alpha-\beta$. Because of these conditions on $\alpha^{\prime}, \beta^{\prime}$ in (6), (7) we must have $0<\alpha-\beta<1, \alpha-\beta<\frac{1}{2}\left(v-\gamma+\frac{3}{2}\right)$. Then (6), (7) become

$$
\begin{gathered}
\int_{0}^{\infty} \xi^{-\alpha-\beta} \frac{R_{v-\gamma-\alpha+\beta+1, v}(t ; \xi, a)}{J_{v}^{2}(\xi a)+Y_{v}^{2}(\xi a)} \psi(\xi) d \xi=\frac{2^{\alpha-\beta}}{\Gamma(1+\beta-\alpha)} t^{-v+\gamma+\alpha-\beta-1} \int_{a}^{t} x^{1+v-\gamma}\left(t^{2}-x^{2}\right)^{\beta-\alpha} g_{1}(x) d x \\
+\frac{2^{v-\gamma+\alpha-\beta+1}}{\pi \Gamma(\beta-\alpha+1)} \Gamma(v-\gamma+1) \int_{0}^{\infty} u^{-\alpha-\beta} \frac{S_{-v+\gamma-\alpha+\beta, v-\gamma-\alpha+\beta+1}(t u)}{J_{v}^{2}(u a)+Y_{v}^{2}(u a)} J_{v}(u a) \psi(u) d u \\
+\frac{2^{\alpha-\beta}}{\Gamma(1+\beta-\alpha)} t^{-v+\gamma+\alpha-\beta-1} \int_{0}^{\infty} \frac{u^{-2 \alpha+1} \psi(u)}{J_{v}^{2}(u a)+Y_{v}^{2}(u a)}\left\{\int_{0}^{a} \rho^{1+v-\gamma}\left(t^{2}-\rho^{2}\right)^{\beta-\alpha} R_{v-\gamma, v}(\rho ; u, a) d \rho\right\} d u, \\
a \leq t \leq c,
\end{gathered}
$$

and

$$
\begin{gathered}
\int_{0}^{\infty} \xi^{1-\alpha-\beta} \frac{R_{v-\gamma-\alpha+\beta, v}(t ; \xi, a)}{J_{v}^{2}(\xi a)+Y_{v}^{2}(\xi a)} \psi(\xi) d \xi=\frac{2^{1-\alpha+\beta}}{\Gamma(\alpha-\beta)} t^{v-\gamma-\alpha+\beta} \int_{1}^{\infty} x^{1-v+\gamma}\left(x^{2}-t^{2}\right)^{\alpha-\beta-1} g_{2}(x) d x, \\
c<t<\infty .
\end{gathered}
$$

Now we multiply both sides of (12) by $t^{\nu-\gamma-\alpha+\beta+1}$ and differentiate with respect to $t$ and then using the relation

$$
\frac{1}{t} \frac{d}{d t}\left[t^{\mu} R_{\mu, v}(t ; \xi, a)\right]=t^{\mu-1} \xi R_{\mu-1, v}(t ; \xi, a),
$$

we obtain

$$
\begin{aligned}
& \int_{0}^{\infty} \xi^{1-\alpha-\beta} \frac{R_{v-\gamma-\alpha+\beta, v}(t ; \xi, a)}{J_{v}^{2}(\xi a)+Y_{v}^{2}(\xi a)} \psi(\xi) d \xi=\frac{2^{\alpha-\beta}}{\Gamma(1+\beta-\alpha)} t^{-v+\gamma+\alpha-\beta-1} \frac{d}{d t} \int_{a}^{1} x^{1+v-\gamma}\left(t^{2}-x^{2}\right)^{\beta-\alpha} g_{1}(x) d x \\
& +\frac{2^{v-\gamma+\alpha-\beta+1}}{\pi \Gamma(1+\beta-\alpha)} \Gamma(v-\gamma+1) t^{-v+\gamma+\alpha-\beta-1} \frac{d}{d t}\left\{t^{v-\gamma-\alpha+\beta+1}\right. \\
& \left.\quad \int_{0}^{\infty} u^{-\alpha-\beta} \frac{S_{-v+\gamma-\alpha+\beta, v-\gamma-\alpha+\beta+1}(t u)}{J_{v}^{2}(u a)+Y_{v}^{2}(u a)} J_{v}(u a) \psi(u) d u\right\} \\
& +\frac{2^{\alpha-\beta}}{\Gamma(1+\beta-\alpha)} t^{-v+\gamma+\alpha-\beta-1} \frac{d}{d t} \int_{0}^{\infty} \frac{u^{-2 \alpha+1} \psi(u)}{J_{v}^{2}(u a)+Y_{v}^{2}(u a)} \\
& \left\{\int_{0}^{a} \rho^{\left.1+v-\gamma\left(t^{2}-\rho^{2}\right)^{\beta-\alpha} R_{v-\gamma, v}(\rho ; u, a) d \rho\right\} d u,}\right. \\
& a \leq t \leq c .
\end{aligned}
$$

As before, equations (13) and (14) have identically the same left-hand side which is therefore defined for all $t \geq a$. Since we have assumed $\gamma+\alpha-\beta$ to be a positive integer and $\alpha-\beta<\frac{1}{2}\left(v-2 \gamma+\frac{3}{2}\right)$, therefore by the lemma in section 2 , we find 


$$
\begin{aligned}
\psi(\xi)= & \xi^{\alpha+\beta}\left[\frac{2^{\alpha-\beta}}{\Gamma(1-\alpha+\beta)} \int_{a}^{c} t^{-v+\gamma+\alpha-\beta} R_{v-\gamma-\alpha+\beta, v}(t ; \xi, a) \overline{G_{1}}(t) d t\right. \\
& +\frac{2^{1-\alpha+\beta}}{\Gamma(\alpha-\beta)} \int_{c}^{\infty} t^{1+\nu-\gamma+\alpha+\beta} R_{v-\gamma-\alpha+\beta, v}(t ; \xi, a) \overline{G_{2}}(t) d t \\
& \left.+\frac{2^{\alpha-\beta}}{\Gamma(1-\alpha+\beta)} \int_{0}^{\infty} u^{-\alpha-\beta} K(u, \xi) \psi(u) d u\right] .
\end{aligned}
$$

where

$$
\begin{aligned}
\overline{G_{1}}(t) & =\frac{d}{d t} \int_{a}^{t} x^{1+v-\gamma}\left(t^{2}-x^{2}\right)^{\beta-\alpha} g_{1}(x) d x, \\
\overline{G_{2}}(t) & =\int_{1}^{\infty} x^{1-v+\gamma}\left(x^{2}-t^{2}\right)^{\alpha-\beta-1} g_{2}(x) d x, \\
\bar{K}(u, \xi) & =\frac{\left(\frac{1}{\pi}\right) 2^{1+v-\gamma} \Gamma(1+v-\gamma) J_{v}(u a) \overline{F_{1}}(u, \xi)+u^{1-\alpha+\beta} \overline{F_{2}}(u, \xi)}{J_{v}^{2}(u a)+Y_{v}^{2}(u a)}, \\
\overline{F_{1}}(u, \xi) & =\int_{a}^{c} t^{-v+\gamma+\alpha-\beta} \frac{d}{d t}\left\{t^{v-\gamma-\alpha+\beta-1} S_{-v+\gamma-\alpha+\beta, v-\gamma-\alpha+\beta+1}(t u)\right\} R_{v-\gamma+\beta-\alpha, v}(t ; \xi, a) d t, \\
\overline{F_{2}}(u, \xi) & =\int_{a}^{c} t^{-v+\gamma+\alpha-\beta} \overline{K_{1}}(u, t) R_{v-\gamma+\beta-\alpha, v}(t ; \xi, a) d t,
\end{aligned}
$$

and

$$
\overline{K_{1}}(u, t)=\frac{d}{d t} \int_{0}^{a} \rho^{1+v-\gamma}\left(t^{2}-\rho^{2}\right)^{\beta-\alpha} R_{v-\gamma, v}(\rho ; u, a) d \rho
$$

The equation (15) is a Fredholm integral equation of second kind.

\section{SPECIAL CASES}

(i) If we make $a \rightarrow 0$ and $\nu>0$ with $\lim _{a \rightarrow 0} \frac{\psi(\xi)}{Y_{v}(a \xi)}-A(\xi)$ and $\alpha$ replaced by $(1-\alpha) / 2$ and $\beta$ by $\frac{1}{2}$ and putting $v-\gamma=\mu$, the dual integral equations (4) and (5) become

$$
\begin{gathered}
\int_{0}^{\infty} \xi^{\alpha} A(\xi) J_{\mu}(x \xi) d \xi=g_{1}(x), \quad a \leq x \leq c, \\
\int_{0}^{\infty} A(\xi) J_{\mu}(x \xi) d \xi=g_{2}(x), \quad x \geq c .
\end{gathered}
$$

These dual integral equations were considered by Noble [4]. From (11) and (15), the solution of the above dual integral equations become:

$$
\begin{gathered}
\text { For } 0<\alpha<2, \quad \mu>\frac{1}{2}-\alpha \\
A(\xi)=\frac{(2 \xi)^{1-\frac{1}{2} \alpha}}{\Gamma\left(\frac{1}{2} \alpha\right)} \int_{0}^{c} t^{-\mu-\frac{1}{2} \alpha+1} J_{\mu+\frac{1}{2} \alpha}(t \xi) G_{1}(t) d t+\frac{2^{\frac{1}{2} \alpha} \xi^{1-\frac{1}{2} \alpha}}{\Gamma\left(1-\frac{1}{2} \alpha\right)} \int_{0}^{\infty} t^{\mu+\frac{1}{2} \alpha} J_{\mu+\frac{1}{2} \alpha}(t \xi) G_{2}(t) d t,
\end{gathered}
$$


where

$$
\begin{aligned}
& G_{1}(t)=\int_{0}^{t} g_{1}(x) x^{1+\mu}\left(t^{2}-x^{2}\right)^{-1+\frac{1}{2} \alpha} d x, \\
& G_{2}(t)=-\frac{d}{d t} \int_{1}^{\infty} g_{2}(x) x^{1-\mu}\left(x^{2}-t^{2}\right)^{-\frac{1}{2} \alpha} d x,
\end{aligned}
$$

and for $-2<\alpha<0, \mu>-\frac{3}{2}-\alpha$

$$
A(\xi)=\frac{2^{-\frac{1}{2} \alpha} \xi^{-\frac{1}{2} \alpha}}{\Gamma\left(1+\frac{1}{2} \alpha\right)} \int_{0}^{c} t^{-\mu-\frac{1}{2} \alpha} J_{\mu+\frac{1}{2} \alpha}(t \xi) \overline{G_{1}}(t) d t+\frac{2^{1+\frac{1}{2} \alpha} \xi^{1-\frac{1}{2} \alpha}}{\Gamma\left(-\frac{1}{2} \alpha\right)} \int_{c}^{\infty} t^{\mu+\frac{1}{2} \alpha+1} J_{\mu+\frac{1}{2} \alpha}(t \xi) \overline{G_{2}}(t) d t,
$$

where

$$
\begin{aligned}
& \overline{G_{1}}(t)=\frac{d}{d t} \int_{0}^{t} g_{1}(x) x^{1+\mu}\left(t^{2}-x^{2}\right)^{\frac{1}{2} \alpha} d x, \\
& \overline{G_{2}}(t)=\int_{1}^{\infty} g_{2}(x) x^{1-\mu}\left(x^{2}-t^{2}\right)^{-1-\frac{1}{2} \alpha} d x .
\end{aligned}
$$

This result was obtained in [4] directly.

(ii) Let $\nu=1, \gamma=\frac{1}{2}, \alpha=\frac{1}{2}$ and $\beta=0$. Then from (4), (5), the system of equations

$$
\begin{aligned}
W_{\frac{1}{2^{1}}}^{-1}\left[\xi^{-1} \psi(\xi) ; x\right]-g_{1}(x), & a \leq x \leq c \\
W_{\frac{1}{z^{1}}}^{-1}[\psi(\xi) ; x]-g_{2}(x), & c<x<\infty
\end{aligned}
$$

reduce to a Fredholm integral equation of second kind given by, from equation (15),

$$
\psi(\xi)=\left(\frac{2 \xi}{\pi}\right)^{\frac{1}{2}}\left[\int_{a}^{c} R_{0,1}(t ; \xi, a) \overline{G_{1}}(t) d t+\int_{c}^{\infty} t R_{0,1}(t ; \xi, a) \overline{G_{2}}(t) d t+\int_{0}^{\infty} u^{\frac{1}{2}} \bar{K}(u, \xi) \psi(u) d u\right]
$$

where

$$
\begin{aligned}
& \overline{G_{1}}(t)=\frac{d}{d t} \int_{a}^{t} x^{\frac{3}{2}}\left(t^{2}-x^{2}\right)^{-\frac{1}{2}} g_{1}(x) d x, \\
& \overline{G_{2}}(t)=\int_{t}^{\infty} x^{\frac{1}{2}}\left(x^{2}-t^{2}\right)^{-\frac{1}{2}} g_{2}(x) d x, \\
& \bar{K}(u, \xi)=\frac{\left(\frac{2}{x}\right)^{\frac{1}{2}} J_{1}(u a) \overline{F_{1}}(u, \xi)+u^{\frac{1}{2}} \overline{F_{2}}(u, \xi)}{J_{v}^{2}(u a)+Y_{v}^{2}(u a)} \\
& \overline{F_{1}}(u, \xi)=\int_{a}^{c} R_{0,1}(t ; \xi, a) \frac{d}{d t}\left\{t^{-1} S_{-1,1}(t u)\right\} d t, \\
& \overline{F_{2}}(u, \xi)=\int_{a}^{c} \overline{K_{1}}(u, t) R_{0,1}(t ; \xi, a) d t,
\end{aligned}
$$

and 


$$
\overline{K_{1}}(u, t)=\frac{d}{d t} \int_{0}^{a} \rho^{\frac{3}{2}}\left(t^{2}-\rho^{2}\right)^{-\frac{1}{2}} R_{\frac{1}{2}, 1}(\rho ; u, a) d \rho
$$

with

$$
S_{\mu, v}(z)=z^{\mu+1} \int_{0}^{\infty} t e^{-1 z} F\left(\frac{1}{2}-\frac{1}{2} \mu+\frac{1}{2} v, \frac{1}{2}-\frac{1}{2} \mu-\frac{1}{2} v ; \frac{3}{2} ;-t^{2}\right) d t,
$$

$z>0, F(a, b ; c ; x)$ being the hypergeometric function, and

$$
R_{\frac{1}{\mathfrak{2}^{\prime}}}(\rho ; u, a)=\left(\frac{2}{\pi \rho u}\right)^{\frac{1}{2}}\left[\sin \rho u Y_{1}(u a)+\cos \rho u J_{1}(u a)\right] .
$$

(iii) When $v=\gamma=\frac{1}{2}, \alpha-\beta=\frac{1}{2}$, then

$$
\begin{array}{ll}
W_{0, \frac{1}{2}}^{-1}\left[\xi^{-2 \alpha} \psi(\xi) ; x\right]=g_{1}(x), & a \leq x \leq c \\
W_{0, \frac{1}{2}}^{-1}\left[\xi^{-2 \beta} \psi(\xi) ; x\right]=g_{2}(x), & c<x<\infty
\end{array}
$$

reduce to a Fredholm integral equation of second kind given by, from equation (15),

$\psi(\xi)=\left(\frac{2}{\pi}\right)^{\frac{1}{2}} \xi^{\alpha+\beta}\left[\int_{a}^{c} t^{\frac{1}{2}} R_{-\frac{1}{2^{\prime}} \frac{1}{2}}(t ; \xi, a) \overline{G_{1}}(t) d t+\int_{c}^{\infty} t^{\frac{1}{2}} R_{-\frac{1}{2} \frac{1}{2}}(t ; \xi, a) \overline{G_{2}}(t) d t+\int_{0}^{\infty} u^{-\alpha-\beta} \bar{K}(u, \xi) \psi(u) d u\right]$,

where

and

$$
\begin{aligned}
\overline{G_{1}}(t) & =\frac{d}{d t} \int_{a}^{t} x\left(t^{2}-x^{2}\right)^{-\frac{1}{2}} g_{1}(x) d x, \\
\overline{G_{2}}(t) & =\int_{1}^{\infty} x\left(x^{2}-t^{2}\right)^{-\frac{1}{2}} g_{2}(x) d x, \\
\bar{K}(u, \xi) & =\frac{\pi u a}{2}\left[\left(\frac{2}{\pi}\right) J_{\frac{1}{2}}(u a) \overline{F_{1}}(u, \xi)+u^{\frac{1}{2}} \overline{F_{2}}(u, \xi)\right] \\
\overline{F_{1}}(u, \xi) & =\int_{a}^{c} t^{\frac{1}{2}} \frac{d}{d t}\left\{t^{-\frac{3}{2}} S_{-\overline{2}^{\prime}, \frac{1}{2}}(t u)\right\} R_{-\frac{1}{2}, \frac{1}{2}}(t ; \xi ; a) d t, \\
\overline{F_{2}}(u, \xi) & =\int_{a}^{c} t^{\frac{1}{2}} \overline{K_{1}}(u, t) R_{-\frac{1}{2^{2}} \frac{1}{2}}(t ; \xi, a) d t, \\
\overline{K_{1}}(u, t) & =\frac{d}{d t} \int_{0}^{a} \rho\left(t^{2}-\rho^{2}\right)^{-\frac{1}{2}} R_{0, \frac{1}{2}}(\rho ; u, a) d \rho
\end{aligned}
$$

with

$$
\begin{aligned}
& R_{-\frac{1}{2}, \frac{1}{2}}(t ; \xi, a)=-\left(\frac{2}{\pi \xi}\right)(a t)^{-\frac{1}{2}} \cos \xi(t-a), \\
& R_{0, \frac{1}{2}}(t ; u, a)=-\left(\frac{2}{\pi u a}\right)^{\frac{1}{2}}\left[\cos u a J_{0}(u t)+\sin u a Y_{0}(u t)\right] .
\end{aligned}
$$

(iv) Finally, a purely formal case, when $v=0, \gamma \rightarrow 0, \alpha=-\frac{1}{2}$ and $\beta=0$, then 


$$
\begin{aligned}
& W_{0,0}^{-1}[\xi \psi(\xi) ; x]=g_{1}(x), \quad a \leq x \leq c \\
& W_{0,0}^{-1}[\psi(\xi) ; x]=g_{2}(x), \quad c<x<\infty
\end{aligned}
$$

reduce to a Fredholm integral equation of second kind given by, from equation (11),

$$
\psi(\xi)=\left(\frac{2}{\pi \xi}\right)^{\frac{1}{2}}\left[\int_{a}^{c} t^{\frac{1}{2}} R_{\frac{1}{2}, 0}(t ; \xi, a) G_{1}(t) d t+\int_{c}^{\infty} t^{\frac{1}{2}} R_{\frac{1}{2}, 0}(t ; \xi, a) G_{2}(t) d t+\int_{0}^{\infty} u^{\frac{1}{2}} K(u, \xi) \psi(u) d u\right],
$$

where

and

$$
\begin{aligned}
G_{1}(t) & =\int_{a}^{t} x\left(t^{2}-x^{2}\right)^{-\frac{1}{2}} g_{1}(x) d x, \\
G_{2}(t) & =-\frac{d}{d t} \int_{1}^{\infty} x\left(x^{2}-t^{2}\right)^{-\frac{1}{2}} g_{2}(x) d x, \\
K(u, \xi) & =\frac{\left(\frac{2}{\pi}\right) J_{0}(u a) F_{1}(u, \xi)+u^{\frac{1}{2}} F_{2}(u, \xi)}{J_{0}^{2}(u a)+Y_{0}^{2}(u a)}, \\
F_{1}(u, \xi) & =\int_{a}^{c} t S_{-\frac{1}{2^{\prime}}, \frac{1}{2}}(t u) R_{\frac{1}{2}, 0}(t ; \xi, a) d t, \\
F_{2}(u, \xi) & =\int_{a}^{c} t^{\frac{1}{2}} K_{1}(u, t) R_{\frac{1}{2}, 0}(t ; \xi, a) d t, \\
K_{1}(u, t) & =\int_{0}^{a} \rho\left(t^{2}-\rho^{2}\right)^{-\frac{1}{2}} R_{0,0}(\rho ; u, a) d \rho,
\end{aligned}
$$

with

$$
R_{\frac{1}{2}, 0}(t ; \xi, a)=\left(\frac{2}{\pi t \xi}\right)^{\frac{1}{2}}\left[\sin t \xi Y_{0}(a \xi)+\cos t \xi J_{0}(a \xi)\right]
$$

ACKNOWLEDGEMENT. We thank CSIR, New Delhi for the award of a senior fellowship to NM and the referee for pointing out the reference [7] and his comments in revising the paper in the present form.

\section{REFERENCES}

[1] SRIVASTAV, R. P. A pair of dual integral equations involving Bessel functions of the first and second kind, Proc. Edin. Math. Soc. 14 (1964), 149-158.

[2] SRIVASTAV, R. P. An axisymmetric mixed boundary value problem for a half-space with a cylindrical cavity, J. Math. \& Mech. 13 (1964), 385-393.

[3] NASIM, C. Dual integral equations involving Weber-Orr transforms, Internat. J. Math. \& Math. Sci. 14 (1991), 163-176.

[4] NOBLE, B. The solution of Bessel function dual integral equations by a multiplying factor method, Proc. Camb. Phil. Soc. 59 (1963), 351-362.

[5] MANDAL, B. N. A note on Bessel function dual integral equation with weight function, Internat. J. Math. \& Math. Sci. 11 (1988), 543-550.

[6] ERDELYI, A. et al. Tables of Integral Transforms II, McGraw-Hill, New York (1954).

[7] NASIM, C. Associated Weber integral transforms of arbitrary order, Ind. J. Pure and Appl. Math. 20 (11), Nov. 1989, 1126-1138. 


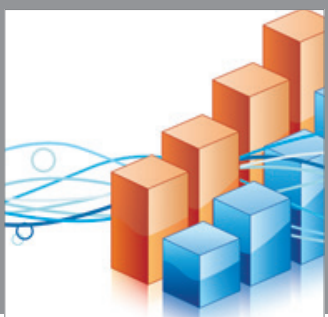

Advances in

Operations Research

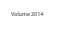

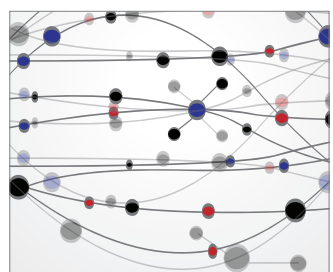

\section{The Scientific} World Journal
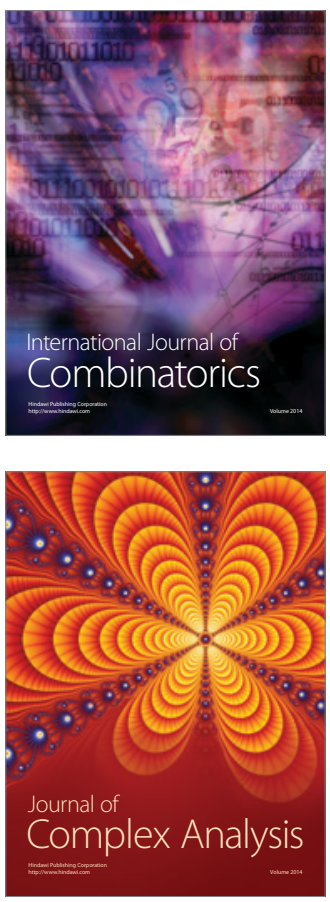

International Journal of

Mathematics and

Mathematical

Sciences
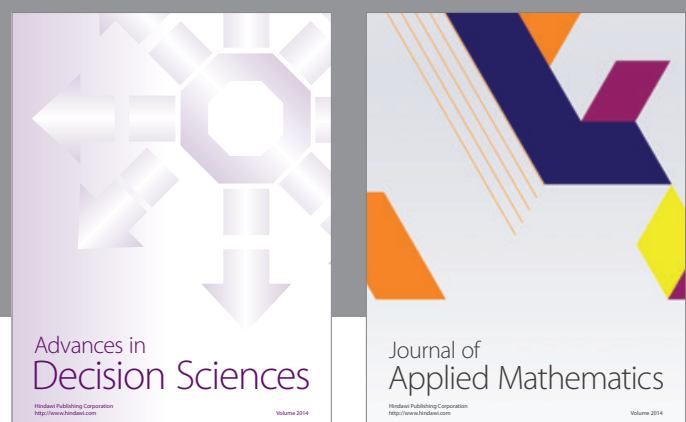

Journal of

Applied Mathematics
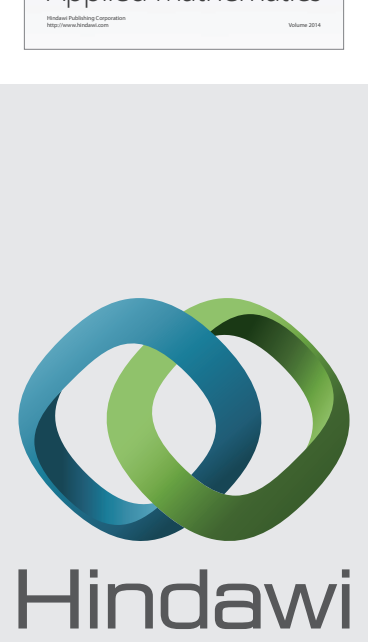

Submit your manuscripts at http://www.hindawi.com
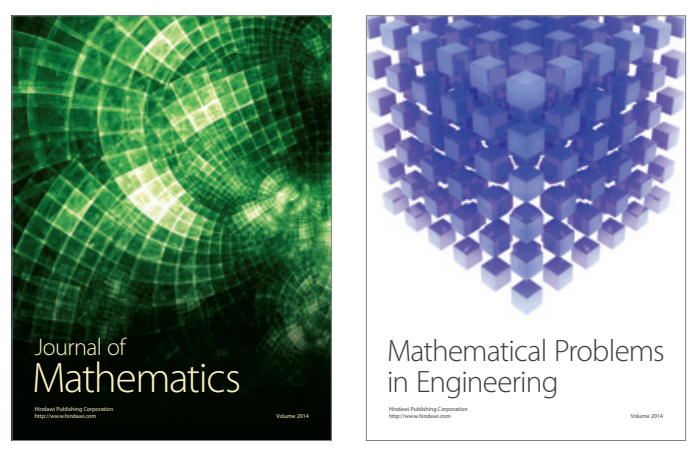

Mathematical Problems in Engineering
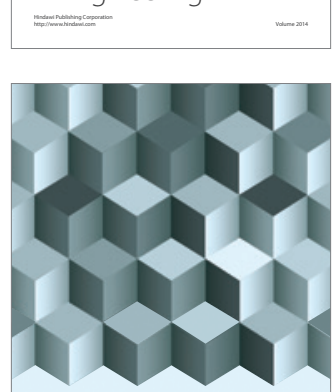

Journal of

Function Spaces
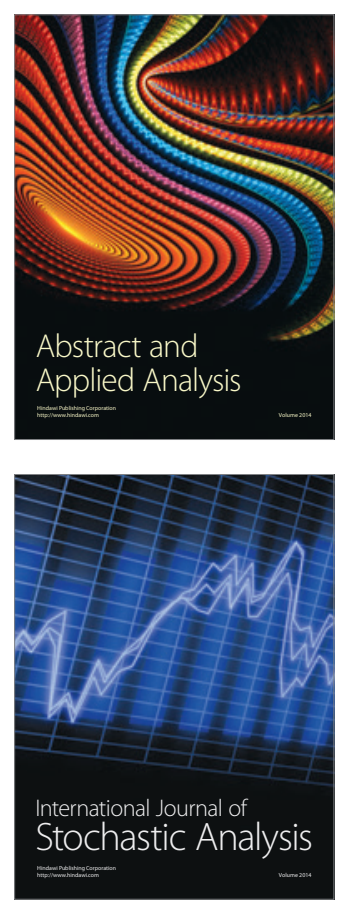

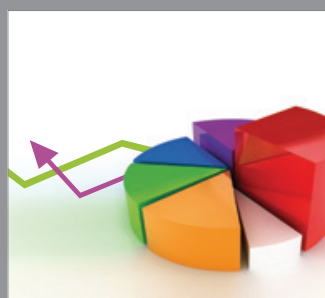

ournal of

Probability and Statistics

Promensencen
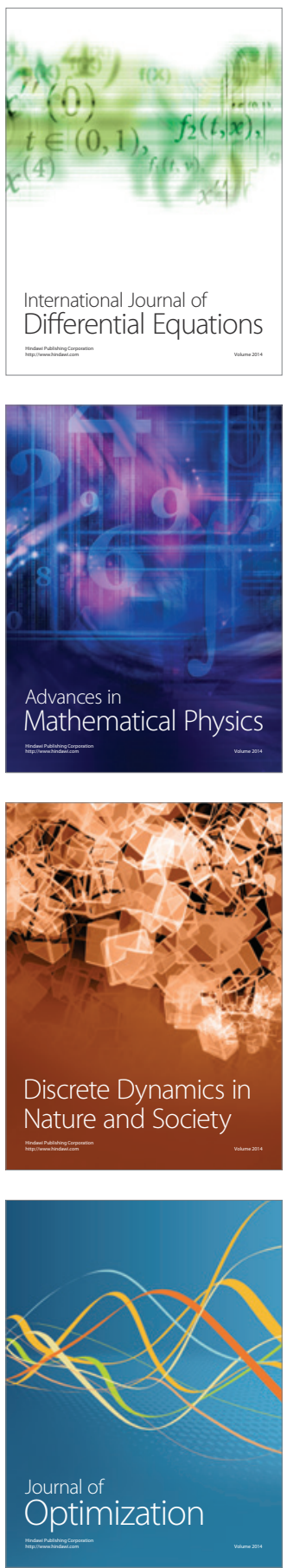